\title{
Fully magnetized plasma flow in a magnetic nozzle
}

\author{
Mario Merino ${ }^{\text {a) }}$ and Eduardo Ahedo ${ }^{\text {b) }}$ \\ Equipo de Propulsión Espacial y Plasmas (EP2), Universidad Carlos III de Madrid, Leganés, Spain
}

(Received 8 December 2015; accepted 2 February 2016; published online 18 February 2016)

\begin{abstract}
A model of the expansion of a plasma in a magnetic nozzle in the full magnetization limit is presented. The fully magnetized and the unmagnetized-ions limits are compared, recovering the whole range of variability in plasma properties, thrust, and plume efficiency, and revealing the differences in the physics of the two cases. The fully magnetized model is the natural limit of the general, 2D, two-fluid model of Ahedo and Merino [Phys. Plasmas 17, 073501 (2010)], and it is proposed as an analytical, conservative estimator of the propulsive figures of merit of partially magnetized plasma expansions in the near region of the magnetic nozzle. (C) 2016 AIP Publishing LLC. [http://dx.doi.org/10.1063/1.4941975]
\end{abstract}

\section{INTRODUCTION}

From the inception of the magnetic nozzle ${ }^{1}(\mathrm{MN})$, there has been a growing interest in their capability to accelerate a plasma "contactlessly" in advanced electric propulsion. Examples of next-generation plasma thrusters based on MNs that are actively being developed include the Helicon Plasma Thruster $^{2,3}$ (HPT), the Electron-Cyclotron-Resonance thruster $^{4,5}$ (ECRT), the Applied-field MPD thruster $^{6}$ (AF-MPDT), and the Variable Specific Impulse Rocket (VASIMR). ${ }^{7}$ In the usual design, a MN consists in an axisymmetric, divergent magnetic field that guides the supersonic expansion of a hot plasma jet. The quasineutral plasma gains axial kinetic energy at the expense of its internal energy, thanks to the self-consistent ambipolar electric field. The reaction to the magnetic forces that shape the plasma expansion is felt on the magnetic circuit of the thruster as "magnetic thrust." MNs are also a topic of research in the field of advanced plasma material processing, ${ }^{8}$ where their non-propulsive use allows to control the flux of plasma to the substrate.

In a previous work, ${ }^{9}$ the DIMAGNO model of the plasma expansion in a divergent $\mathrm{MN}$ was presented. This $2 \mathrm{D}$ two-fluid model treats electrons as a fully magnetized species, whereas heavier ions, on the other hand, are allowed to have arbitrary magnetization. The method of characteristics is used to integrate the hyperbolic equations of supersonic ions. DIMAGNO was instrumental to study the acceleration mechanisms and forces on the plasma, ${ }^{9,10}$ the development of electric currents and electric fields, and the downstream detachment of the plasma from the MN. ${ }^{11-13}$ This model has revealed the richness of $2 \mathrm{D}$ phenomena in the expansion, which are unaccessible to paraxial 1D models. The DIMAGNO model was also used to investigate the conditions for the formation of double-layer structures ${ }^{14}$ and the effects of electron cooling and ion thermal energy. ${ }^{15}$

While the finite ion magnetization regimes have already been analyzed, the fully magnetized ions limit (FMIL) remains to be explored. There are two reasons for this: first,

\footnotetext{
${ }^{a)}$ Electronic mail: mario.merino@uc3m.es and mario.merino@ep2.uc3m.es

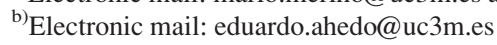

finite ion magnetization covers all cases of practical interest, as extremely high magnetic fields are required to keep ions magnetized into the far downstream expansion region; and second, the FMIL is not a regular limit of the DIMAGNO formulation. In this limit, the character of ion equations degenerates as the Mach characteristic lines no longer carry any useful information, thus requiring a different integration approach. Nonetheless, being able to compare the unmagnetized-ions limit (UMIL) and the FMIL would provide valuable insight on the physics of the $\mathrm{MN}$, as they are the extremes of the operation range of the device.

Following this motivation, the paper presents and discusses the FMIL of the 2D fluid formulation of Ref. 9. Contrary to the finite ion-magnetization case, the FMIL results in a set of completely algebraic equations and thus is simple and fast to compute. The UMIL and the FMIL are then compared to quantify the influence of the level of ion magnetization on the 2D plasma expansion and the propulsive figures of merit of the MN. In particular, it is shown that the thrust gain is rather independent of the ion magnetization degree in the near expansion region, and that the FMIL yields a conservative value of the thrust gain and the plume efficiency for all possible ion magnetizations. In view of this result, the 2D FMIL solution is proposed for the quick estimation of the propulsive performance of the MN in the general case.

In this regard, there are several models in the literature that present some degree of similarity with the FMIL model presented here. First, Andersen et al. ${ }^{1}$ applied the wellknown paraxial (i.e., quasi-1D) model of the expansion of a gas in a channel of slowly varying area to a MN, providing algebraic expressions for the axial profiles of the mean ion velocity and plasma density. This model coincides with the radially averaged equations of the DIMAGNO model and can be used to estimate thrust with good accuracy within the near expansion of the MN. However, the radial variability of plasma properties, the development of electric currents in the plasma, plume efficiency, and plasma detachment cannot be computed with a quasi-1D model. Second, Fruchtman et $a l .{ }^{16}$ also studied the paraxial approximation. Beyond reproducing the solution of Andersen et al., they postulated a 
self-similar expansion of the plasma density radial profile in an attempt to recover some of the radial features of the expansion and derive a formula for the magnetic thrust. Third, Little and Choueiri ${ }^{17}$ took the 2D DIMAGNO model of Ref. 9 as their starting point and applied several simplifying assumptions in order to derive an approximate, "less numerical" solution which can be used for quick computations of the plasma response and the propulsive figures of merit.

The rest of the paper is structured as follows. Section II derives the FMIL model from the general fluid equations and briefly discusses the numerical solution. Section III explores and compares the plasma properties and propulsive performance at the UMIL and FMIL. Section IV discusses the applicability and limitations of the FMIL to describe plasma expansions with finite ion magnetizations and comments further on the differences with the works in the literature enumerated above. Finally, conclusions of this work are gathered in Section V.

\section{2D MODEL OF THE FULLY MAGNETIZED PLASMA EXPANSION}

The derivation of the FMIL model commences with the complete 2D equations of the full two-fluid model of Ref. 9. Where not specified, the conventional notation defined therein will be used here too. The model describes the steady-state expansion of hot electrons, " $e$," and singlecharged cold ions, " $i$," in an externally applied magnetic field, $\boldsymbol{B}$. The plasma is assumed to be quasineutral $\left(n_{i} \simeq n_{e} \equiv n\right)$, collisionless and low-beta (i.e., negligible induced magnetic field effects), and to have a clean lateral boundary with vacuum. For simplicity, electrons will be treated as an isotropic, isothermal species so that $T_{e}=$ const, with $T_{e}=p_{e} / n$, although other thermodynamic models are available. ${ }^{14,15}$ Furthermore, electrons are assumed to be fully magnetized and to have negligible inertia according to the following scaling: $u_{e} \ll \sqrt{T_{e} / m_{e}} \ll e B R / m_{e}$, where $R$ is the initial plasma tube radius that serves as the macroscopic length of the problem. Note that the last ordering states that $\ell_{e}=\sqrt{T_{e} m_{e}} /(e B) \ll R$, where $\ell_{e}$ is the electron Larmor radius. Under these hypotheses, the continuity and momentum equations for ions and electrons read:

$$
\begin{gathered}
\nabla \cdot\left(n \boldsymbol{u}_{i}\right)=0 ; \quad \nabla \cdot\left(n \boldsymbol{u}_{e}\right)=0, \\
m_{i}\left(\boldsymbol{u}_{i} \cdot \nabla\right) \boldsymbol{u}_{i}=-e \nabla \phi+e \boldsymbol{u}_{i} \times \boldsymbol{B}, \\
0=-T_{e} \nabla \ln \left(n / n_{0}\right)+e \nabla \phi-e \boldsymbol{u}_{e} \times \boldsymbol{B} .
\end{gathered}
$$

The equations above can be normalized with $R, m_{i}, e$, and $T_{e}$. Likewise, we may normalize density with a reference value $n_{0}$, e.g., its value at the center of the throat. The isothermal sound velocity of the plasma $c_{s}=\sqrt{T_{e} / m_{i}}$ and the longitudinal ion Mach number $M=\sqrt{u_{z i}^{2}+u_{r i}^{2}} / c_{s}$ are defined in the usual way.

As shown in Ref. 9, the electron equations can be directly integrated into algebraic expressions. In particular, the azimuthal projection of Eq. (3) indicates that the electron streamtubes coincide with the magnetic streamtubes, with $u_{\perp e}=0$. Then, the parallel and perpendicular components of Eq. (3) provide two conservation laws along electron streamtubes

$$
\begin{gathered}
T_{e} \ln \frac{n}{n_{0}}-e \phi=H_{e}(\psi) ; \\
e u_{\theta e} B=-T_{e} \frac{\partial \ln \left(n / n_{0}\right)}{\partial \mathbf{1}_{\perp}}+e \frac{\partial \phi}{\partial \mathbf{1}_{\perp}} \\
\equiv-\frac{\partial H_{e}}{\partial \mathbf{1}_{\perp}}=-r B \frac{d H_{e}}{d \psi},
\end{gathered}
$$

where $\psi$ is the streamfunction of the applied magnetic field (i.e., $\nabla \psi=r B \mathbf{1}_{\perp}$ ), and $-H_{e}(\psi) / e$ is the thermalized potential of each streamtube. Finally, using the solenoidal character on the $\boldsymbol{B}$ field $(\nabla \cdot \boldsymbol{B}=0)$, the electron continuity equation in Eq. (1) can be written as a third conservation law along $\psi$

$$
n u_{\| e} / B=G_{e}(\psi)
$$

with $G_{e}(\psi)$ being the electron-to-magnetic flux ratio at each streamtube.

From the four scalar ion equations, however, only the first integrals associated to the conservation of canonical angular momentum and mechanical energy along ion streamtubes (which can differ from the electron/magnetic streamtubes) can be found, ${ }^{9}$ and in general, a numerical scheme must be employed to integrate the ion motion in the meridional plane. The method of characteristics was chosen in Ref. 9, which exploits the fact that physical information is carried only along the characteristic lines in the hyperbolic problem of the supersonic expansion of ions: while the ion streamlines transmit information on ion mechanical energy and canonical momentum, the Mach lines convey information on the electric potential and electron pressure.

The level of ion magnetization is measured by the nondimensional ion gyrofrequency parameter at center of the throat section, $\hat{\Omega}_{i 0}=e B_{0} R / \sqrt{T_{e} m_{i}}$. The general model of Ref. 9 admits any finite value of $\hat{\Omega}_{i 0}$. The FMIL corresponds to taking $\hat{\Omega}_{i 0} \rightarrow \infty$ and presents a number of profound consequences on the structure of the equations and the physics of the expansion.

First, as indicated by the azimuthal ion equation, ions are now fully attached to the magnetic lines, with $u_{\perp i}=0$ just like the electrons before. Moreover, comparing the advective and the magnetic term in Eq. (2), $u_{\theta i} \sim M^{2} \hat{\Omega}_{i 0}^{-1} c_{S}$, so $u_{\theta i}$ vanishes in the limit $\Omega_{i 0} \rightarrow \infty$. Observe that, in the absence of initial ion swirl $\left(u_{\theta i}=0\right.$ at $\left.z=0\right)$, the ion azimuthal current $j_{\theta i}$ is also zero in the whole plasma domain in the UMIL, since ion separation does not induce any $u_{\theta i}$ when $B \rightarrow 0$ in that case. Hence, only when $\hat{\Omega}_{i 0}$ is finite and nonzero $u_{\theta i}>0$ develops downstream, and therefore, a maximal $u_{\theta i}$ exists for some value of $\hat{\Omega}_{i 0}$. This value of $u_{\theta i}$ is small in all cases of practical interest. ${ }^{9}$ Second, from Eq. (5), the azimuthal electron velocity scales as $u_{\theta e} \propto \hat{\Omega}_{i 0}^{-1} c_{s}$, so this drift can also be neglected in first approximation in the FMIL. Hence, ions and electrons move along magnetic lines with a velocity $O\left(c_{S}\right)$ and one may simply write 


$$
\boldsymbol{u}_{i}=u_{i} \mathbf{1}_{\|} ; \quad \boldsymbol{u}_{e}=u_{e} \mathbf{1}_{\|}
$$

where $\mathbf{1}_{\|}=\boldsymbol{B} / B$. Third, like with electrons, it is now possible to reduce the ion continuity equation in Eq. (1) to

$$
n u_{i} / B=G_{i}(\psi),
$$

and the parallel projection of Eq. (2) yields again the equation of ion mechanical energy, which is conserved along the ion/electron/magnetic streamtubes

$$
\frac{1}{2} m_{i} u_{i}^{2}+e \phi=H_{i}(\psi)
$$

Finally, the perpendicular component of Eq. (2) in terms of the magnetic field curvature, $\kappa_{B}$, provides an expression for the magnetic force on ions

$$
e u_{\theta i} B=\kappa_{B} m_{i} u_{i}^{2}+e \frac{\partial \phi}{\partial 1_{\perp}} .
$$

In summary, we have 4 algebraic equations (Eqs. (4), (6), (8), and (9)) for 4 unknowns $\left(u_{i}, u_{e}, n, \phi\right)$, with 4 integration functions of $\psi\left(H_{e}, G_{e}, G_{i}, H_{i}\right)$ that are calculated from the initial (i.e., throat) conditions. Given these and the geometry of the external magnetic field, these equations can be readily solved to obtain directly the full 2D plasma response. In fact, the evolution of these plasma properties along each streamline $\psi=$ const is completely independent from neighboring lines, and therefore, the flow field can be solved in a line-by-line basis, regardless of the complexity of the geometry of the MN. For this reason, it is convenient to express the solution as a function of $\psi$ and a new independent variable $\lambda=B(z, \psi) / B(0, \psi)$, i.e., the magnetic field on each streamline normalized with its value upstream, $B(0, \psi)$. For instance, solving for $u_{i}(\lambda, \psi)$ one finds

$$
\frac{1}{2} m_{i}\left[u_{i}^{2}(\lambda, \psi)-u_{i}^{2}(0, \psi)\right]-T_{e} \ln \frac{u_{i}(\lambda, \psi)}{u_{i}(0, \psi)}=-T_{e} \ln \lambda .
$$

This equation is indeed the exact $2 \mathrm{D}$ version of the equivalent 1D equation proposed by Andersen et al. and later by Fruchtman et al. Once $u_{i}(\lambda, \psi)$ is known, the solutions for $\phi$, $n$, and $u_{e}$ in terms of $(\lambda, \psi)$ are straightforward. The solution of the 2D plasma expansion is therefore equivalent to solving a $1 \mathrm{D}$ expansion for each streamline.

Observe that when $u_{i}(0, \psi)=$ const, i.e., when the ion velocity is uniform at the throat, $u_{i}(\lambda, \psi)$ becomes only a function of $\lambda$. As a consequence, $n(\lambda, \psi) / n(0, \psi)$ and $\phi(\lambda, \psi)-\phi(0, \psi)$ are also only functions of $\lambda$. In other words, the 2D evolution of these plasma variables depends only on the relative drop of magnetic field along each line, and the plasma profiles are self-similar over $\lambda=$ const surfaces. These convex surfaces are not normal to the magnetic streamlines in general and are shown in Fig. 1 in Section III. Note also that geometric self-similarity over $z=\mathrm{const}$ planes (used in the derivation of the model of Fruchtman et al.) is therefore not strictly satisfied neither in the FMIL nor for any finite value of $\hat{\Omega}_{i 0}$.

As a last (and uncoupled) step, Eqs. (5) and (10) yield the magnetic force terms, $u_{\theta e} B$ and $u_{\theta i} B$. Note that, while $u_{\theta e}$ and $u_{\theta i}$ go to zero in the FMIL, these magnetic forces are finite. Indeed, the magnetic force on electrons depends essentially on the electron pressure $n T_{e}$, and the magnetic force on ions on the ion inertia and the curvature of the magnetic lines.

The streamline independence of the FMIL contrasts with the finite-ion-magnetization case, where each point influences the whole domain within its downstream Mach cone. Mathematically, in the FMIL the Mach lines have disappeared, leaving only the streamlines as characteristic lines. Therefore, the problem degenerates into a singlecharacteristic hyperbolic one in this limit. Physically, this agrees with the negligible role of the perpendicular pressure and electric field terms with respect to the magnetic ones when $\hat{\Omega}_{i 0} \rightarrow \infty$; in a finite-ion-magnetization case, these pressure terms are responsible for carrying the information in the Mach directions.

Observe also that since the FMIL turns the plasma expansion into a single-characteristic hyperbolic problem even when ions are subsonic, it is easy to compute the subsonic convergent part of the $\mathrm{MN}$, a task that required a separate numerical method in the finite-ion-magnetization case.

\section{RESULTS}

To analyze the differences between the FMIL and UMIL, this section compares the expansion of a given plasma under the two limits. The $\hat{\Omega}_{i 0} \rightarrow \infty$ limit is computed solving the equations described above, whereas the $\hat{\Omega}_{i 0} \rightarrow 0$ limit is integrated with the DIMAGNO code. ${ }^{9}$ For the sake of illustration, the magnetic field of a single current loop of radius $R_{L}=3.5 R$ located at $z=0$ is used. For this particular $\mathrm{MN}$, the turning point (i.e., the location where the outermost magnetic line carrying plasma turns around) is located at about $z=16 R$ and $r=23 R$. The following radial profile for density, potential, and velocity is imposed in the two cases at the magnetic throat $(z=0)$ between $r=0$ and $R$

$$
\begin{aligned}
& n=n_{0} \exp \left(-a r^{2} / R^{2}\right) ; \quad \phi=0, \\
& u_{z i}=u_{z e}=1.01 c_{s} ; \quad u_{r i}=u_{r e}=0,
\end{aligned}
$$

where $a$ is a parameter that controls the shape of the profile ( $a=3 \ln 10$ is chosen here), and the 1.01 value is to ensure supersonic conditions for the method of characteristics in the UMIL case. Moreover, an electron theta-pinch equilibrium and no ion swirl are enforced at the throat

$$
e u_{\theta e} B=2 a T_{e} r / R^{2} ; \quad u_{\theta i}=0 .
$$

The values of $n, \phi, u_{i}$, and $u_{e}$ change monotonously with $\hat{\Omega}_{i 0}$, so the FMIL and UMIL cover their full range of variability. The results of the two simulations are displayed in Fig. 1, and the major differences between the two ion magnetization limits are described below. In all cases, the variations grow downstream:

1. The radial plasma density profile is less focused around the axis the higher $\hat{\Omega}_{i 0}$ is, as seen in Fig. 1(a). The effect is large already at $x=15 R$, where the density difference at the periphery is about 2 orders of magnitude. It is 
(a)

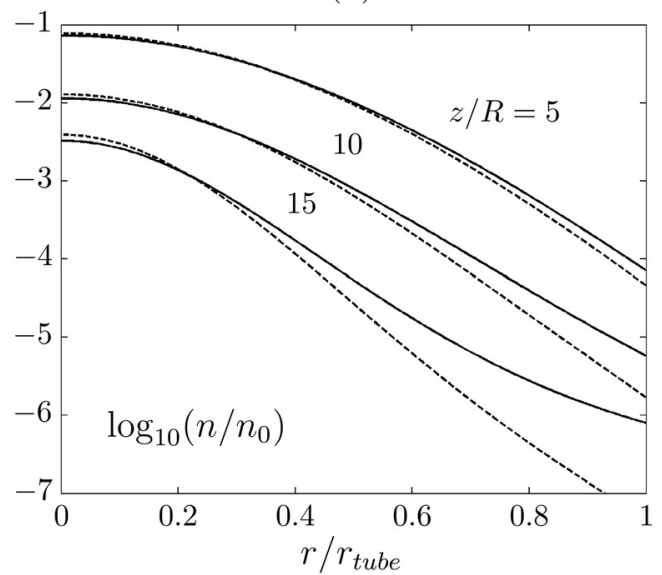

(c)

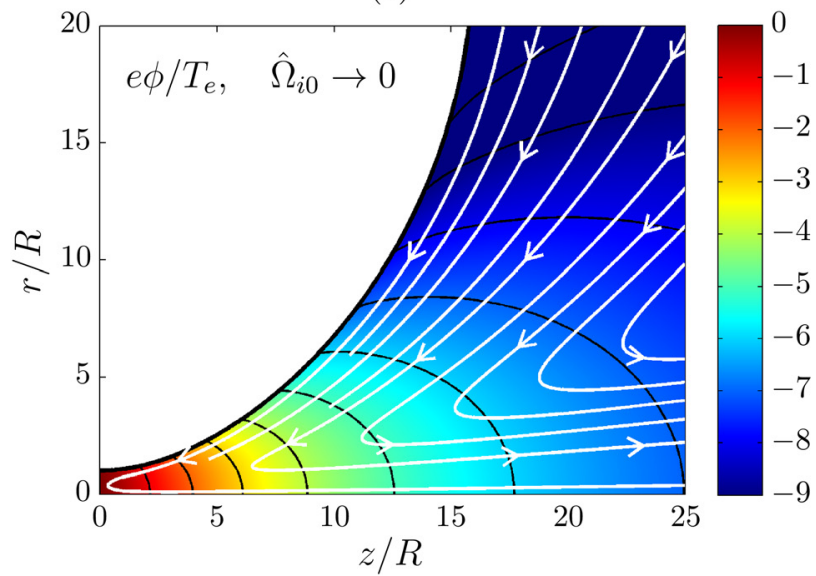

(b)

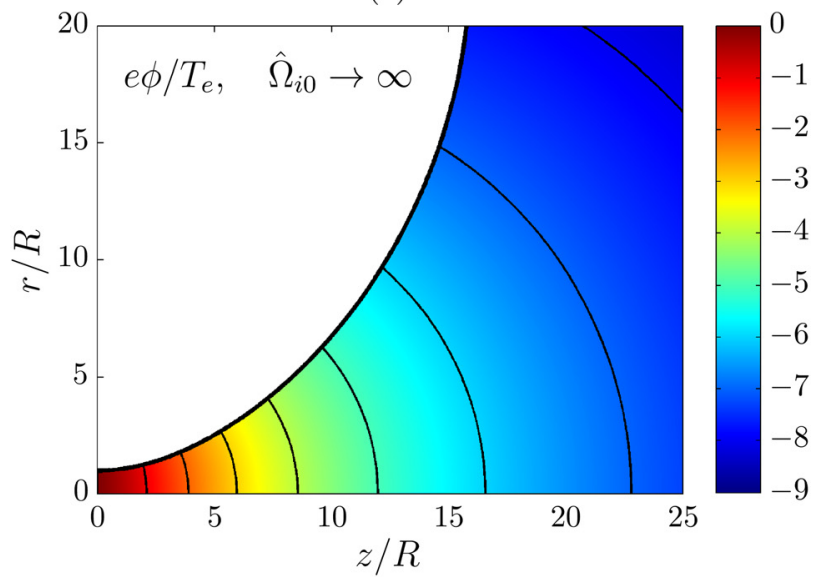

(d)

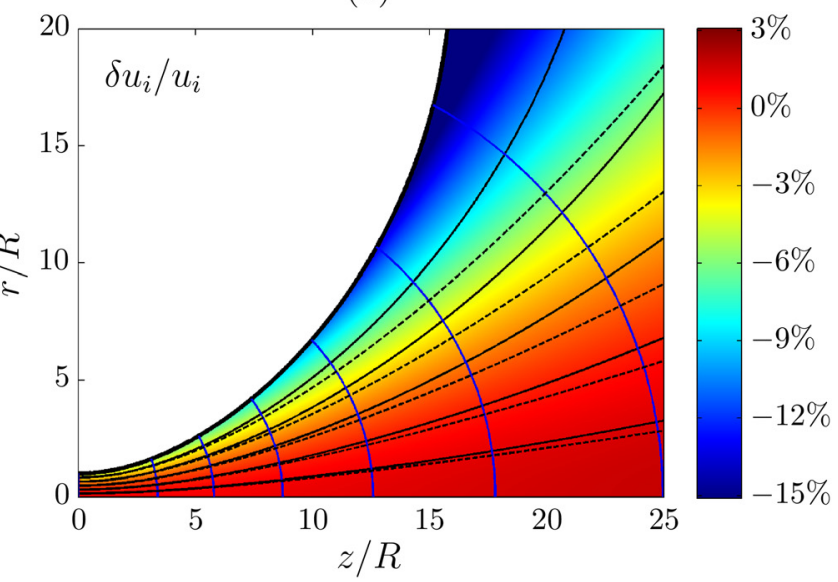

FIG. 1. Comparison of the FMIL and UMIL simulations. Figure (a) displays the radial density profile at various values of $z$. Here, $r_{t u b e}$ is the radius of the last plasma tube at each $z=$ const section. Solid lines denote the FMIL and dashed lines the UMIL. Figures (b) and (c) display the ambipolar electric potential in the UMIL and FMIL cases, respectively. Isopotential curves, spaced in increments of $e \delta \phi / T_{e}=1$, are shown as black lines. The arrowed white lines of Figure (c) show the longitudinal electric currents that develop in the plasma, which become of the order of half the ion flux downstream. ${ }^{9}$ Figure (d) shows the relative difference in ion velocity, $\delta u_{i} / u_{i}=\left(u_{i, F}-u_{i, U}\right) / u_{i, F}$, where subindices $F$ and $U$ denote the FMIL and UMIL, respectively. Ion streamlines have been drawn in solid line for the FMIL and in dashed line for the UMIL. The blue lines that intersect the MN axis are $\lambda=$ const lines.

stressed that the plasma density is not geometrically selfsimilar over $z=$ const planes in either case.

2. Since $\phi$ is merely a function of $n$ on each electron streamline (Eq. (4)), the ambipolar electric potential $\phi$ largely differs between the UMIL and FMIL, too. The direction of the electric field changes as well, from highly perpendicular in the UMIL to essentially parallel in the FMIL, as can be inferred from the isopotential lines of Figs. 1(b) and 1(c). Observe that in the FMIL the isopotential lines coincide with the self-similarity lines $\lambda=$ const, which are shown in Fig. 1(c).

3. An ion velocity difference of about $15 \%$ between the two limits occurs at the plasma periphery, with the FMIL displaying a lower velocity there. Since $u_{i}$ grows with $\sqrt{-2 e \phi / m_{i}}$ (Eq. (9)), this is in agreement with the reduced rarefaction and lower potential drop observed above. Figure 1(d) shows also that ion velocity at the axis is slightly larger in the FMIL.

4. The direction of the ion flow changes substantially as depicted in Fig. 1(d). While the UMIL ions separate soon from the magnetic lines and therefore diverge less, the FMIL ions are fully attached and no separation occurs as a consequence of the FMIL model assumptions themselves. Indeed, this phenomenon is the primary cause for the differences in density and velocity profiles observed in points (1) and (3).

5. No separation means that no longitudinal electric currents form in the plasma in the FMIL. In contrast, in the UMIL local currents exist even if the plasma jet is globally current free, ${ }^{9}$ as can be seen in Fig. 1(c). The condition $j_{z}=j_{r}=0$ is referred to as local current ambipolarity and is generally invoked a priori in ambipolar diffusion models. The initial conditions of Eq. (12) suffice to guarantee local current ambipolarity in the whole plasma domain in the FMIL but not for finite values of $\hat{\Omega}_{i 0}$. The formation of longitudinal (meridian) currents is indeed a common occurrence in axisymmetric plasmas with finite ion magnetization. While in the present collisionless model the currents are caused by the divergent magnetic geometry, they have been found also for cylindrical, collisional plasmas in a uniform magnetic field. ${ }^{18}$

6. Evidently, since there is no ion separation in the FMIL, plasma detachment does not occur, and ions return along the magnetic lines, canceling thrust. In contrast, plasma 
detachment is guaranteed in finite-ion-magnetization cases, thanks to the growing ion inertia and the decreasing magnetic strength. In fact, very large values of $\hat{\Omega}_{i 0}$ are required to keep ions attached beyond the MN turning point; as an example, a plasma with $\hat{\Omega}_{i 0}=200$ has a downstream detachment behavior closer to the UMIL than the FMIL. ${ }^{13}$

These observations indicate that the $2 \mathrm{D}$ character of the plasma in the MN changes considerably already in the near region of the expansion when $\hat{\Omega}_{i 0} \rightarrow \infty$ with respect to the cases with finite $\hat{\Omega}_{i 0}$. It is worth explaining these differences from the viewpoint of the perpendicular balance of forces in the plasma. In a low-ion-magnetization plasma (and in general, in finite $\hat{\Omega}_{i 0}$ cases sufficiently far downstream), the magnetic force on ions is negligible, and the dominant forces on them are electric. These forces are in charge of opening them radially to maintain quasineutrality in the whole domain, and from this condition comes the necessary radial drop in density to create the self-consistent radial electric field. Ion deflection in this case is seen to be insufficient to match the magnetic lines downstream, ${ }^{13}$ and ion separation occurs. On the other hand, in the FMIL ion deflection is guaranteed by the magnetic force. Indeed, in this limit deflection is perfect, in the sense that ions are forbidden to separate at all from the turning magnetic lines. The perpendicular electric field becomes irrelevant and exists only when there is a differential drop in plasma density between neighboring lines due to the expansion itself.

Note that in all cases, however, the longitudinal force that accelerates ions downstream is electric, associated to the ambipolar potential drop that develops along the expansion. Therefore, the plasma acceleration mechanism still relies on the ambipolar electric field which scales as the electron temperature, $T_{e}$.

The diamagnetic axial force $-e u_{\theta e} B_{r}$ is essential in the operation of the $\mathrm{MN}$, as the associated reaction force (which is felt on the MN generator) is the magnetic thrust. ${ }^{9,11}$ The ion axial magnetic force $e u_{\theta i} B_{r}$ is however paramagnetic and causes drag (i.e., negative thrust). Actually, the thrust function $F(z)$, which indicates the thrust force generated up to at a $z=$ const section (denoted as $S_{z}$ ), can be computed as the integral of the total plasma momentum that traverses that section, or equivalently, as initial thrust at the throat $F_{0}=$ $F(0)$ plus the integral of the total axial magnetic force on the plasma in the volume $\mathcal{V}_{z}$ delimited by the throat and $S_{z}$ (see
Eqs. (42) and (43) of Ref. 9). In other words, assuming $B_{r}>$ 0 in the divergent $\mathrm{MN}$ without loss of generality

$$
F(z)=2 \pi \int_{S_{z}} n\left(T_{e}+m_{i} u_{z i}^{2}\right) r \mathrm{~d} r=F_{0}-\int_{\mathcal{V}_{z}} j_{\theta} B_{r} d \mathcal{V}
$$

where the plasma pressure at the plasma-vacuum boundary has been assumed negligible. Using Eqs. (4), (5), (9), and (10), it is possible to obtain a compact expression for the thrust function in the UMIL $(U)$ and the FMIL $(F)$ cases

$$
\begin{gathered}
F_{U}(z)=F_{0}-\int_{\mathcal{V}_{z}} T_{e} \frac{\partial n}{\partial \mathbf{1}_{\perp}} \sin \alpha_{B} d \mathcal{V}, \\
F_{F}(z)=F_{0}-\int_{\mathcal{V}_{z}}\left(T_{e} \frac{\partial n}{\partial \mathbf{1}_{\perp}}+n m_{i} \kappa_{B} u_{i}^{2}\right) \sin \alpha_{B} d \mathcal{V},
\end{gathered}
$$

where $\sin \alpha_{B}=B_{r} / B$. Comparing these two formulas, it is clear that the electron contribution produces positive magnetic thrust in both cases, whereas ions cause a negative contribution in the FMIL as expected. As a side note, this ion contribution is missed in the model of Fruchtman et al., where the $m_{i} \kappa_{i} u_{i}^{2}$ term in their Eq. (2) (our Eq. (10)) is not included (an aspect inherited from Ref. 19). Since this is the dominant term in the perpendicular momentum equation of the inertia-driven, supersonically divergent ions, this oversight also changes the character of the ion swirl motion, from essentially zero (due to the conservation of ion angular canonical momentum around the axis ${ }^{9}$ ) to a pure $E \times B$ drift in their model.

Likewise, the plume efficiency function $\eta_{\text {plume }}(z)$ defined in Ref. 9, which accounts for divergence losses, can be calculated as follows:

$$
\eta_{\text {plume }}(z)=\int_{S_{z}} n u_{z i}^{3} r \mathrm{~d} r / \int_{S_{z}} n u_{i}^{2} u_{z i} r \mathrm{~d} r .
$$

The thrust gain $F(z) / F_{0}$ and $\eta_{\text {plume }}(z)$ are shown in Fig. 2 for the FMIL and UMIL simulations.

The most outstanding aspect of Fig. 2(a) is that the thrust gain $F / F_{0}$ in the near region expansion depends only weakly on the value of $\hat{\Omega}_{i 0}$. While a higher ion magnetization means a lower thrust gain, the differences are modest before the turning point, and only become apparent further downstream. Indeed, the negative ion contribution of Eq. (16) is small in the near expansion region, but dominates downstream where $\alpha_{B}$ is large, and partially cancels thrust in (a)

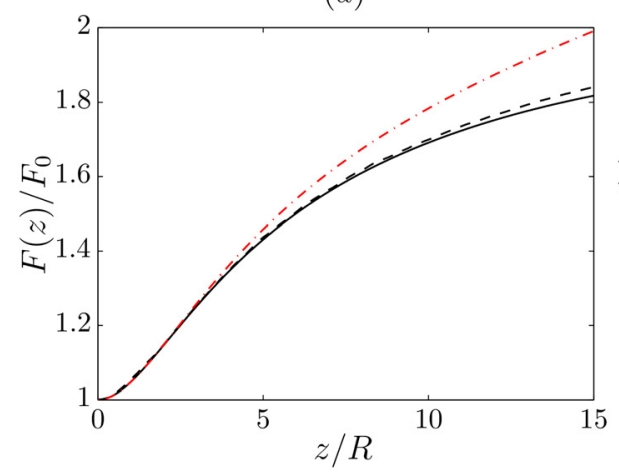

(b)

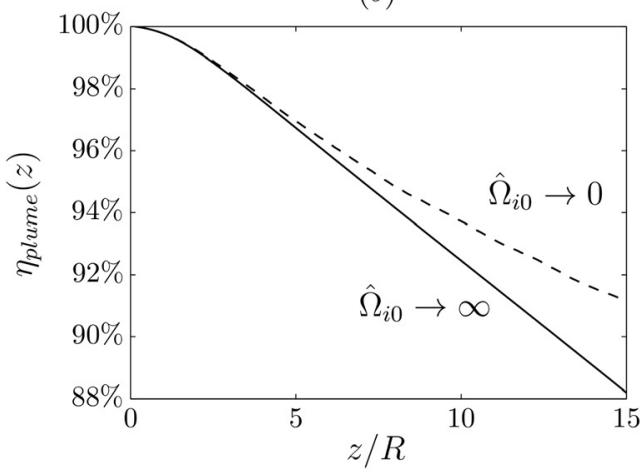

FIG. 2. Thrust gain function $F(z) / F_{0}$ (a) and plume efficiency function $\eta_{\text {plume }}(z)$ (b) in the FMIL (solid lines) and the UMIL (dashed lines). Thrust predicted by the radially averaged, paraxial 1D model based on the value of $B / B_{0}$ at the axis is shown in (a) as a red dashed-dotted line. 
the FMIL. The similarity of $F / F_{0}$ in the two limits is also due to the axial ambipolar acceleration of ions being essentially unaffected by the ion magnetization in the near expansion, as discussed above.

In fact, the thrust function is successfully approximated by $1 \mathrm{D}$ models in the near region for this very reason. It can be shown that for an isothermal plasma, the 1D model of Andersen et al. ${ }^{1}$ leads after some manipulation to the following formula of thrust gain as a function of the Mach number:

$$
\frac{F}{F_{0}}=\frac{M^{2}+1}{2 M},
$$

and $M$ is related to the area expansion ratio $\left(B_{0} / B\right.$ in a $1 \mathrm{D}$ model) by Eq. (11) applied at the axis. The 1D thrust gain is plotted on Fig. 2(a) for comparison. Visibly, this model provides an optimistic value of $F / F_{0}$ with respect to the two 2D simulations. This is partly due to the $1 \mathrm{D}$ model ignoring the radial or divergence losses in the expansion, accounted for by $\eta_{\text {plume }}$ (a purely $2 \mathrm{D}$ result), and also due to the plasma profiles not being self-similar over $z=$ const surfaces, a condition upon which the radially averaging of plasma profiles in 1D models is based.

Regarding the plume efficiency in Fig. 2(b), it is again observed that a higher $\hat{\Omega}_{i 0}$ yields a lower $\eta_{\text {plume }}$, as could have been anticipated by the analysis of the ion streamline deflection above. A high value of this figure of merit is strongly related to the successful detachment of the plasma from the MN, which is hindered by a large $\hat{\Omega}_{i 0}$. Finally, it should be noted that the $\hat{\Omega}_{i 0} \rightarrow \infty$ and $\hat{\Omega}_{i 0} \rightarrow 0$ constitute, respectively, the lower and upper limits for both the thrust gain and the plume efficiency at each $z$ for a given plasma.

\section{DISCUSSION}

For a quick estimation of MN performance and for preliminary thruster design, a simple model of the plasma expansion in the near region of a given magnetic field is useful. While the FMIL model is of course only strictly applicable when $\hat{\Omega}_{i 0} \rightarrow \infty$, it is an excellent candidate for those tasks given its mathematical simplicity, physical consistency, and its capability to provide acceptable (and conservative) estimates of the thrust gain $F / F_{0}(z)$ and the plume efficiency $\eta_{\text {plume }}(z)$ regardless of the actual value of $\hat{\Omega}_{i 0}$. Indeed, in the example simulations of Section III, the differences in $F / F_{0}$ and $\eta_{\text {plume }}$ between the UMIL and FMIL are smaller than $2 \%$ and $4 \%$, respectively, at $z=15 R$. The plasma density $n$ and the ion velocity $u_{i}$ can also be estimated in this way for finite values of $\hat{\Omega}_{i 0}$, although a higher error is committed near the periphery of the plasma. Naturally, the magnitude of the error depends on the actual value of $\hat{\Omega}_{i 0}$ and on how far downstream the FMIL model is used.

Notwithstanding this, there are several limitations that should be taken into account when applying the FMIL solution to finite- $\hat{\Omega}_{i 0}$ cases. First and foremost, errors in all variables become large beyond the region of the turning point, where ion separation is substantial, and usage of the model in that region should be avoided unless $\hat{\Omega}_{i 0}$ is sufficiently high. The conditions for ion separation derived in Ref. 13 can help to decide upon the applicability of the FMIL solution at a given $z=$ const section. Second, the model does not recover the radial relative focalization of the plasma density around the axis observed in finite $\hat{\Omega}_{i 0}$ cases. ${ }^{9}$ Third, the potential map $\phi$ obtained in the $\hat{\Omega}_{i 0} \rightarrow \infty$ limit is an inadequate substitute for that of a low- $\hat{\Omega}_{i 0}$ case, where the perpendicular electric field is much larger and becomes a fundamental feature of the expansion due to its central role in ion deflection and ion separation. Fourth, the plasma electric currents are zero in the FMIL. In the case of the electron azimuthal current, the product $-e u_{\theta e} B$ varies little with $\hat{\Omega}_{i 0}$ and can be used to approximate other cases. To recover the ion magnetic force and the plasma currents in finite-ion-magnetization cases, the full 2D fluid model is required. Finally but fundamentally, the FMIL model cannot be used to study plasma detachment in finite- $\hat{\Omega}_{i 0}$ cases, which is one of the crucial processes that takes place in a propulsive $\mathrm{MN}$.

To conclude this section, we further comment on the differences between this exact 2D FMIL solution and the approximate MN model of Little and Choueiri, ${ }^{17}$ who derived an approximate solution of the 2D model of Ref. 9, with the goal of finding a more algebraic and thus quicker solution. However, the set of expressions they obtain are not compact and simple, and their derivation employs a set of conflicting hypotheses: On the one hand, the model assumes a priori that ion velocity is parallel to $\boldsymbol{B}$ and forces local current ambipolarity in the flow (i.e., as in the $\hat{\Omega}_{i 0} \rightarrow \infty$ limit). On the other hand, the magnetic force on ions is deemed negligible (i.e., as in the limit $\hat{\Omega}_{i 0} \rightarrow 0$ ). As a result, the electric field alone must create the required normal force to deflection streamlines so they artificially match the magnetic streamsurfaces. The electric field calculated in this way is largely overestimated and yields an incorrect electric potential $\phi$. The next consequence is that density, computed from $\phi$, does not fulfill the continuity equation in their solution, and ad-hoc correcting factors for $\phi, n$, and $M$ must be added to recover mass conservation and match the solution of Ref. 9 .

\section{CONCLUSIONS}

This paper has explored the fully-magnetized-ions limit of the plasma expansion in a magnetic nozzle, based on the $\hat{\Omega}_{i 0} \rightarrow \infty$ limit of the general 2D fluid model of Ref. 9. In this limit, ion and electron streamlines coincide with the magnetic lines and no information is transmitted along Mach lines. The 2D model becomes fully analytical and can be solved in a line-by-line basis, with completely algebraic conservation equations.

The comparison of the FMIL with the unmagnetizedions case has revealed the complete range of variability of plasma properties for a given $\mathrm{MN}$ and plasma conditions at the throat. Variations grow downstream and, in particular, near the plasma periphery; they become large in the neighborhood of the MN turning point and beyond. Electric currents go to zero in the FMIL, and the nature of ion deflection changes from electrically driven to magnetically driven, but the longitudinal ambipolar electric field is still the main longitudinal ion acceleration mechanism. 
Interestingly, the differences in thrust and plume efficiency functions between the $\hat{\Omega}_{i 0} \rightarrow \infty$ and $\hat{\Omega}_{i 0} \rightarrow 0$ are small in the near expansion. This allows one to use the FMIL solution to yield a conservative approximation of the propulsive figures of merit in finite- $\hat{\Omega}_{i 0}$ cases. Therefore, the analytical FMIL model is proposed as a simple model for fast MN calculations. The limitations of the model for this task have been discussed; in particular, it cannot be used to study downstream plasma detachment-a task that requires the full 2D fluid model. The model compares favorably in terms of simplicity and consistency with previous, related works in the literature.

Finally, it is worth noting that the 2D FMIL model opens the way to study fully magnetized plasma expansions in 3D magnetic nozzles. This is of particular interest in the study of plasma jet magnetic deflection, which can enable contactless thrust vector control with a steerable MN. ${ }^{20}$

\section{ACKNOWLEDGMENTS}

This work has been supported by the Spanish $R \& D$ National Plan, Grant No. ESP2013-41052-P. A preliminary version of this work was presented in Ref. 21.

${ }^{1}$ S. Andersen, V. Jensen, P. Nielsen, and N. D'Angelo, Phys. Fluids 12, 557-560 (1969).

${ }^{2}$ O. Batishchev, IEEE Trans. Plasma Sci. 37, 1563-1571 (2009).

${ }^{3}$ M. Merino, J. Navarro, S. Casado, E. Ahedo, V. Gómez, M. Ruiz, E. Bosch, and J. G. del Amo, "Design and development of a $1 \mathrm{~kW}$-class helicon antenna thruster," in 34th International Electric Propulsion Conference, IEPC-2015-297 (Electric Rocket Propulsion Society, Fairview Park, OH, 2015).

${ }^{4}$ J. C. Sercel, "Electron-cyclotron-resonance (ECR) plasma acceleration," in AIAA 19th Fluid Dynamics, Plasma Dynamics and Lasers Conference, 1987.

${ }^{5}$ J. Jarrige, P. Q. Elias, F. Cannat, and D. Packan, "Characterization of a coaxial ECR plasma thruster," in 44th AIAA Plasmadynamics and Lasers Conference, San Diego, 2013.

${ }^{6}$ G. Krülle, M. Auweter-Kurtz, and A. Sasoh, J. Propul. Power 14, 754-763 (1998)

${ }^{7}$ C. Diaz, Sci. Am. 283, 90-97 (2000).

${ }^{8}$ K. Schoenberg, R. Gerwin, R. Moses, J. Scheuer, and H. Wagner, Phys. Plasmas 5, 2090-2104 (1998).

${ }^{9}$ E. Ahedo and M. Merino, Phys. Plasmas 17, 073501 (2010).

${ }^{10}$ M. Merino and E. Ahedo, IEEE Trans. Plasma Sci. 39, 2938-2939 (2011).

${ }^{11}$ E. Ahedo and M. Merino, Phys. Plasmas 18, 053504 (2011).

${ }^{12}$ E. Ahedo and M. Merino, Phys. Plasmas 19, 083501 (2012).

${ }^{13}$ M. Merino and E. Ahedo, Plasma Sources Sci. Technol. 23, 032001 (2014).

${ }^{14}$ M. Merino and E. Ahedo, Phys. Plasmas 20, 023502 (2013).

${ }^{15}$ M. Merino and E. Ahedo, IEEE Trans. Plasma Sci. 43, 244-251 (2015).

${ }^{16}$ A. Fruchtman, K. Takahashi, C. Charles, and R. Boswell, Phys. Plasmas 19, 033507 (2012).

${ }^{17}$ J. M. Little and E. Y. Choueiri, Phys. Plasmas 20, 103501 (2013).

${ }^{18}$ I. Kaganovich, V. Rozhansky, L. Tsendin, and I. Veselova, Plasma Sources Sci. Technol. 5, 743 (1996).

${ }^{19}$ K. Takahashi, T. Lafleur, C. Charles, P. Alexander, and R. Boswell, Phys. Rev. Lett. 107, 235001 (2011).

${ }^{20}$ M. Merino and E. Ahedo, "Towards thrust vector control with a 3D steerable magnetic nozzle," in 34th International Electric Propulsion Conference (Electric Rocket Propulsion Society, Fairview Park, OH, 2015).

${ }^{21}$ M. Merino and E. Ahedo, "Modelling the expansion of magnetized plasma jets in electric propulsion," in 32nd International Conference on Phenomena in Ionized Gases TL19 (European Physical Society, 2015). 